\title{
Mediating Role of Rumination and Negative Affect in the Effect of Mind-Wandering on Symptoms in Patients With Obsessive-Compulsive Disorder
}

\author{
Pengchong Wang ${ }^{1,2 t}$, Wenwen Cao ${ }^{1,2 t}$, Tao Chen ${ }^{3,4}$, Jian Gao ${ }^{1,2}$, Yifan Liu ${ }^{1,2}$, \\ Xiangyun Yang ${ }^{1,2}$, Fanqiang Meng ${ }^{1,2}$, Jing Sun ${ }^{5 *}$ and Zhanjiang $\mathrm{Li}^{1,2 *}$
}

${ }^{1}$ Beijing Key Laboratory of Mental Disorders, National Clinical Research Centre for Mental Disorders, Beijing Anding Hospital, Capital Medical University, Beijing, China, ${ }^{2}$ Advanced Innovation Center for Human Brain Protection, Capital Medical University, Beijing, China, ${ }^{3}$ Brain and Mind Center, The University of Sydney, Sydney, NSW, Australia, ${ }^{4}$ School of Psychology, The University of Sydney, Sydney, NSW, Australia, ${ }^{5}$ Menzies Health Institute Queensland and School of Medicine and Dentistry, Griffith University, Brisbane, QLD, Australia

To explore the relationship between negative affect, mind-wandering, rumination and obsessive-compulsive symptoms, 100 patients with obsessive-compulsive disorder and 100 healthy controls were assessed using the Obsessive-Compulsive Inventory, the Beck Anxiety Inventory, the Beck Depression Inventory, the Mind Wandering Scale and the Ruminative Response Scale. The results show that (i) patients diagnosed with obsessive-compulsive disorder displayed higher obsessive-compulsive symptoms, negative affect, mind-wandering and rumination compared with healthy controls; (ii) negative affect, mind-wandering and rumination were positively correlated with the severity of obsessive-compulsive symptoms; (iii) mind-wandering predicted the severity of obsessive-compulsive symptoms (both directly and indirectly); (iv) rumination and negative affect mediated the relationship between mind-wandering and obsessive-compulsive symptoms. The results preliminarily reveal the relationship between mind-wandering and psychopathological obsessive-compulsive symptoms, providing a reference for exploring novel psychological treatments for obsessive-compulsive disorder.

Keywords: obsessive-compulsive disorder, mind wandering, rumination, negative affect, mediation analysis

\section{INTRODUCTION}

Obsessive-compulsive disorder (OCD) is characterised by frequent and severe unwanted intrusive thoughts and repetitive or ritualised behaviours (1). While some obsessions are related to external factors, OCD involves frequent spontaneous and unwanted thoughts, also known as unintentional mind-wandering (MW), that abruptly into one's consciousness without any identifiable evoking stimuli $(2,3)$. Cognition models of $\operatorname{OCD}(4,5)$ often focus on how thoughts can transform into psychopathological obsessions and mental distress. Individuals who misinterpret or ruminate on normal mental phenomena are more likely to develop obsessive-compulsive symptoms (OCS) (6). While MW has been associated with OCS, the relationship between them is not well understood. 
Dysfunctional MW may be common to multiple mental health disorders (7). The inward focus associated with MW may increase the likelihood of self-absorbed thinking, which can elicit negative thoughts (8). For example, people who frequently engage in unintentional MW are more likely to report symptoms of depression, anxiety and stress (9). Thus, dysfunctional MW may enhance negative thoughts and negative affect, increasing the risk of psychopathological symptoms.

\section{BACKGROUND}

\section{MW and OCD Symptomatology}

According to a dynamic framework of thought (7), MW is a special case of spontaneous thinking that is less constrained and more goal oriented compared with rumination and obsessive thinking. MW is predictive of stress (10), depression (11) and anxiety (12) and has become a prominent topic in cognitive psychology (13). MW also provides a new perspective, namely that spontaneous thoughts may play a role in the cause and maintenance of OCS (14). MW is closely related to OCS in nonclinical samples $(6,14-16)$. For example, Seli, Risko, Purdon and Smilek (14) found that MW is correlated with different dimensions of OCS. In addition, MW is considered a specific type of out-of-context thought. Fradkin and Huppert (2) found that OCS is associated with a higher probability of recalling negative (v. non-negative) out-of-context thoughts, implying that MW may be a risk factor for OCS. However, to date, few studies have explored how the wandering mind affects OCS in clinical samples.

\section{Mediating Effect of Rumination and Negative Affect in the Relationship Between MW and OCS}

Although some studies have found that MW is correlated with OCS, not all spontaneous thoughts are unique to OCD (17). Understanding when spontaneous thoughts are not adaptive may clarify their role in the aetiology of OCD, shedding light on the still unexplained association between psychopathology and risks to mental health.

According to the MW-perseverative cognition continuum hypothesis, when MW loses its adaptivity and becomes rigid and inflexible, it may result in a repetitive spiral of homogeneous negative thoughts (e.g., excessive rumination and low selfesteem) and lead to cognitive vulnerability $(18,19)$. The spontaneous thought model posits that abnormal spontaneous thoughts can reduce cognitive control and turn into rumination (18). MW may also augment repetitive thinking (20). Marchetti, Koster (21) have demonstrated that internally oriented thoughts during rest may encourage a ruminative self-focus, in turn worsening negative mood.

According to the cognition model of OCD, maladaptive rumination may play a role in the onset and maintenance of psychopathological OCS. Recent evidence suggests that people with OCD can experience severe maladaptive rumination, which has been positively correlated with different dimensions of OCS in non-clinical samples $(6,15,22)$. Maladaptive rumination is a key symptom in people with OCD, who feel responsible for its consequences, thus excessively focus on their thoughts (23). Several studies suggest that ruminating on one's intrusive thoughts may result in abnormal appraisals of naturally occurring spontaneous thoughts, resulting in the persistence of negative affect $(6,24,25)$.

Rumination is a complex cognition vulnerability factor that may mediate the relationship between naturally occurring thoughts and OCS. In light of the MW psychopathology hypothesis, we assume that MW in itself a maladaptive process but may become a risk factor for OCS if it manifests as rigid and inflexible rumination, leading to negative appraisals of naturally occurring thoughts, obsessions and distress.

People with OCD generally experience negative affect comorbidities such as anxiety, depression or disgust $(26,27)$. This negative affect is directly related to not only individuals' wellbeing and life satisfaction (28) but also OCS such as repetitive intrusive thoughts (29). Negative affect is a potential obstacle to OCD treatment and may impede a patient's ability to change, augment patient distress and predict worse treatment outcomes (30).

MW is a critical factor in negative affect states. For example, involuntary $\mathrm{MW}$ is highly correlated with symptoms of anxiety and depression $(9,31)$. Several studies have shown that MW may be indirectly associated with negative affect via rumination, which may lead to anxiety and depression through a variety of mechanisms, often referred to as transdiagnostic factors (32, 33). Marchetti, Van de Putte and Koster (11) found that daydreaming can result in depressive symptoms via excessive self-focus and rumination. Smallwood, O'Connor and Heim (34) found that off-task thoughts are more frequently correlated with depression in high ruminators compared with low ruminators. In addition, Marchetti, Koster and De Raedt (21) reported that higher internal focus during resting states predicts increased levels of rumination, resulting in more negative moods. It has been suggested that MW may induce abnormal rumination (11), meaning that people with OCD are more inclined to catastrophise with negative misinterpretations and intrusive thoughts, thus increasing the negative affect experience and further worsening OCS $(24,25)$.

Previous studies on the relationships between OCS, rumination and MW have mostly been limited to nonclinical samples $(2,14,16,35)$. This study aims to explore (i) MW, rumination and negative affect in people with clinical OCD compared with healthy controls (HCs) and (ii) whether rumination and negative affect have a chain mediating effect on the relationship between MW and OCS. We hypothesise that (i) people with OCD will experience a higher frequency of MW and more severe rumination and negative affect compared with HCs and (ii) higher MW will predict more severe rumination and negative affect, in turn predicting more severe OCS. Shedding light on the underlying processes may increase the understanding of conditions that increase the likelihood of MW related to OCS. 


\section{MATERIALS AND METHODS}

\section{Participants}

In total, 100 patients with OCD (55 females and 45 males) were recruited from the outpatient clinics of Beijing Anding Hospital at Capital Medical University. The patients met the following inclusion criteria: (i) OCD diagnosed according to the MiniInternational Neuropsychiatric Interview (MINI) (36); (ii) aged between 18 and 45 years; and (iii) no history of neurological or other major physical disease, Axis I psychiatric disorders (other than OCD), Axis II personality disorders or drug or alcohol abuse. The Yale-Brown Obsessive-compulsive Scale (YBOCS) (37) mean score was $20.01 \pm 7.496$ (cut-off point $=$ 16.0) (obsession subscale $=10.67 \pm 4.454$; compulsion subscale $=9.30 \pm 4.949$ ). Comorbid depression and anxiety symptoms were measured using the Beck Depression Inventory-II (BDI-II) (14.465 \pm 10.806$)$ and Beck Anxiety Inventory (BAI) (13.538 \pm 10.584 ) respectively, with low overall average results. Of the 100 participants, 37 (37\%) had never been prescribed medications or had stopped taking medications for at least 4 weeks before participating in this study.

For the control group, 100 healthy participants (54 females and 46 males) were recruited via advertising and met the same criteria as the OCD group; however, participants in the control group were excluded if they had been diagnosed with an Axis I psychiatric disorder according to the Diagnostic and Statistical Manual of Mental Disorders (fourth edition) (DSM-IV).

Both groups were matched for age $(28.58 \pm 6.365$ years for patients with OCD, $27.98 \pm 5.53$ years for HCs, $t=0.712$ $p=0.478)$, gender $\left(\chi^{2}=0.020, p=0.887\right)$ and education level $\left(\chi^{2}=1.308, p=0.727\right)$.

\section{Procedure}

All participants were voluntary and signed the informed consent form. The study was approved by the Research Ethics Committee of Beijing Anding Hospital, Capital Medical University, Beijing, China (Ethic no. 2019, scientific research no. 02). Participants were individually interviewed by a trained researcher and completed self-reported measures in a quiet area of the neuropsychological laboratory at the Beijing Key Laboratory of Mental Disorders. Each session lasted approximately $45 \mathrm{~min}$.

\section{Measures \\ Diagnosis and OCS}

The researcher used MINI to diagnose OCD and Y-BOCS to evaluate the severity of OCD in participants who met the DSM-IV OCD diagnostic criteria. The Obsessive-Compulsive Inventory-Revised (OCI-R) (38) was used to measure selfreported OCS in the OCD and HC groups (cut-off point $=21$ ). The OCI-R evaluates OCS experienced in the past month on a 5 -point scale $(0=$ not at all; $4=$ extremely). The Chinese version of OCI-R shows good internal consistency (Cronbach's $\alpha=0.84)$ and test-retest reliability $(r=0.96)$ in both clinical and non-clinical samples (39).

\section{Negative Affect}

The BAI was used to measure anxiety in participants. The inventory evaluates the degree to which 21 different symptoms affected participants during the past week on a 4-point Likert scale $(0=$ not at all; $1=$ mild, little trouble; $2=$ moderate, uncomfortable but tolerable; and 3 = severe, can scarcely be endured). The total score is calculated by adding the scores of all and ranges from 0 to 63 points (cut-off score for clinical anxiety is 16) (40). The Chinese version of the BAI has demonstrated excellent internal consistency (Cronbach's $\alpha=0.95$ ) (41).

The Beck Depression Inventory-II (BDI-II) was used to assess depressive symptoms. This is a popular 21-item self-reported measure of the presence and severity of depression symptoms during the past week. The BDI-II is based on a 4-point Likert scale $(0=$ absent to $3=$ severe $)$. The total score is calculated by adding the scores of all and ranges from 0 to 63 points (cut-off score of clinical depression is 18.5) (42). The Chinese version of the BDI-II has demonstrated good internal consistency (Cronbach's $\alpha=0.94$ ) and test-retest coefficients ( $r=0.55)$ (43).

\section{Rumination}

The Ruminative Response Scale (RRS) is an effective and popular method of measuring rumination. Yang, Ling and Xiao (44) produced a Chinese version of the RRS (RRS-C), which shows good internal consistency (Cronbach's $\alpha=0.88$ ) and mean internal item correlation coefficients (ranging from 0.29 to 0.32 ) in depressed adult patients (45). The RRS-C contains 21 items based on a 4 -point scale $(1=$ never, $2=$ sometimes, $3=$ often, 4 =always), which measures the frequency of the given symptoms. The higher the score (from 21 to 84 points), the higher the level of rumination.

\section{MW}

The Mind Wandering Scale (MWS), taken from the Imaginal Processes Inventory (46), contains 12 items that reflect the frequency of MW characteristics in daily life based on a 5-point scale $(1=$ completely inconsistent, $2=$ comparatively inconsistent, 3 = difficult to determine, $4=$ comparatively consistent, $5=$ completely consistent). The total score ranges from 12 to 60 points, with a higher score indicating more frequent MW. Carciofo, Du, Song and Zhang (47) produced a Chinese version of the MWS (MWS-C), which demonstrates good internal reliability (Cronbach's $\alpha=0.851$; test-retest reliability $=0.718$ ).

\section{Statistical Analysis}

IBM SPSS Statistics 26.0 software was used for statistical analysis. All general clinical data were described as mean \pm standard deviation. The independent samples $t$-test and chi-squared test were used to compare the continuous and category variables, respectively, in the OCD and HC groups. To prevent Type I and Type II errors caused by unsatisfied presuppositions in the classic parameter test, the bias-corrected percentile bootstrap method was used to analyse the mediation effect. The mediation model was tested using PROCESS 3.3 (48) with a sample size of 5,000. A bias-corrected non-parametric percentile with a $95 \%$ confidence interval was selected as the sampling method, all $p$-values were two-tailed, and statistical significance was set at 0.05 . 


\section{RESULTS}

\section{Group Difference}

The results of the independent sample $t$-test showed that OCS, negative affect, rumination and MW scores in the OCD group were all significantly higher than those in the HC group (see Table 1).

\section{Correlation Between Negative Affect, MW, Rumination and OCS}

After controlling for gender, age and education level, partial correlation analysis revealed that negative affect, MW, rumination and OCS were significantly and positively correlated (see Table 2).

Chain mediation effects of rumination and negative affect on the relationship between MW and OCS

\section{Collinearity and Normality Test}

A collinearity test was used to prevent multicollinearity among independent variables. OCS was the dependent variable, while age, gender, education, anxiety, depression, MW and rumination were the independent variables. The results show that all tolerance values were $>0.10$ (gender $=0.978$, age $=0.942$, education $=0.966$, depression $=0.301$, anxiety $=0.401$, $\mathrm{MW}$ $=0.578$, rumination $=0.383$ ), and all variance inflation factor values were $<5$ (gender $=1.022$, age $=1.062$, education $=1.036$, depression $=3.319$, anxiety $=2.494, \mathrm{MW}=1.731$, rumination $=$ 2.608). In the normality test, the skewness values of all variables

TABLE 1 | Differences between obsessive-compulsive symptoms, negative affect, mind-wandering and rumination between groups.

\begin{tabular}{lcccc}
\hline & OCD $(\boldsymbol{n}=\mathbf{1 0 0})$ & HC $(\boldsymbol{n}=\mathbf{1 0 0})$ & $\boldsymbol{t}$ & $\boldsymbol{p}$ \\
\hline BAI & $13.538 \pm 10.584$ & $2.160 \pm 3.320$ & 10.258 & $<0.001$ \\
BDI-II & $14.465 \pm 10.806$ & $3.100 \pm 3.948$ & 9.879 & $<0.001$ \\
OCI-R & $21.540 \pm 14.799$ & $2.300 \pm 3.572$ & 12.638 & $<0.001$ \\
MWS-C & $38.530 \pm 10.105$ & $29.600 \pm 8.430$ & 6.786 & $<0.001$ \\
RRS-C & $44.170 \pm 12.635$ & $30.625 \pm 7.150$ & 9.330 & $<0.001$ \\
\hline
\end{tabular}

OCD, obsessive-compulsive disorder; HC, healthy control; BAl, Beck Anxiety Inventory; BDI-II, Beck Depression Inventory-II; OCI-R, Obsessive-Compulsive Inventory-Revised (Chinese version); MWS-C, Mind Wandering Scale-Chinese; RRS-C, Ruminative Response Scale-Chinese.

TABLE 2 | Correlation between negative affect, mind-wandering, rumination and obsessive-compulsive symptoms $(N=200)$.

\begin{tabular}{lcccc}
\hline & OCI-R & MWS-C & RRS-C & BAI \\
\hline MWS-C & $0.544^{\star \star}$ & - & - & - \\
RRS-C & $0.725^{\star \star}$ & $0.615^{\star \star}$ & - & - \\
BAl & $0.652^{\star \star}$ & $0.501^{\star \star}$ & $0.638^{\star \star}$ & - \\
BDI-II & $0.729^{\star *}$ & $0.579^{\star \star}$ & $0.743^{\star *}$ & $0.757^{\star *}$ \\
\hline
\end{tabular}

BAI, Beck Anxiety Inventory; BDI-II, Beck Depression Inventory-II; OCI-R, ObsessiveCompulsive Inventory-Revised (Chinese version); MWS-C, Mind Wandering ScaleChinese RRS-C, Ruminative Response Scale-Chinese; ${ }^{* *} p<0.01$. ranged from 0.097 to 1.610 , while kurtosis values ranged from -0.512 to 2.363 .

\section{Chain Mediation Analysis}

To investigate whether spontaneous thoughts mediated the linear relationship between negative affect and OCS, we followed the PROCESS 3.3 procedure for testing mediator effects. Given that there were two mediators, Model 6 was selected, and gender, age and education levels were included as covariate variables.

First, we established the mediator model for anxiety and OCS. Hierarchical regression analysis (see Table 3 ) showed that age significantly predicted anxiety $(\beta=0.201, p<0.05)$, MW significantly predicted rumination $(\beta=0.727, p<0.001)$ and anxiety $(\beta=0.176, p<0.01)$, and rumination significantly predicted anxiety $(\beta=0.423, p<0.001)$. Age $(\beta=0.286, p<$ $0.05)$, MW ( $\beta=0.175, p<0.05)$, rumination $(\beta=0.570, p<$ $0.001)$ and anxiety $(\beta=0.408, p<0.001)$ all had a significant predictive effect on OCS.

Results of the mediation effect analysis (see Figure 1) showed that MW directly predicted OCS (direct effect $=0.175, p$ $=0.033$ ), and anxiety and rumination partially mediated this relationship (indirect effect $=0.612$ ). Specifically, the mediation effect consisted of three indirect effects:

- Path 1: MW $\rightarrow$ rumination $\rightarrow$ OCS (indirect effect $=0.414$ )

- Path $2: \mathrm{MW} \rightarrow$ anxiety $\rightarrow$ OCS (indirect effect $=0.072$ )

- Path 3: MW $\rightarrow$ rumination $\rightarrow$ anxiety $\rightarrow$ OCS (indirect effect $=0.126)$.

None of the bootstrap 95\% confidence intervals contained 0 , indicating that all three paths had a significant indirect effect.

Next, we established a mediator model for MW and OCS. Hierarchical regression analysis (see Table 4) showed that MW had a significant positive predictive effect on rumination $(\beta$ $=0.727, p<0.001)$; both MW $(\beta=0.194, p<0.001)$ and rumination $(\beta=0.501, p<0.001)$ significantly predicted depression; and age $(\beta=0.338, p<0.01)$, rumination $(\beta=0.453$, $p<0.001)$ and depression $(\beta=0.577, p<0.001)$ significantly predicted OCS.

TABLE 3 | Hierarchical regression analysis between MW and OCS: Rumination and Anxiety as mediators.

\begin{tabular}{|c|c|c|c|c|c|c|}
\hline \multirow[t]{2}{*}{ Variables } & \multicolumn{2}{|c|}{ Rumination } & \multicolumn{2}{|c|}{ Anxiety } & \multicolumn{2}{|c|}{ OCS } \\
\hline & $\beta$ & $t$ & $\beta$ & $t$ & $\beta$ & $t$ \\
\hline Gender & -1.399 & -1.006 & -0.186 & -0.177 & -1.412 & -1.074 \\
\hline Age & -0.105 & -0.893 & 0.201 & $2.267^{\star}$ & 0.286 & $2.543^{\star}$ \\
\hline Education & -0.457 & -0.670 & -0.348 & -0.676 & -0.451 & -0.698 \\
\hline MW & 0.727 & $10.766^{\star \star \star}$ & 0.176 & $2.744^{\star \star}$ & 0.175 & $2.141^{*}$ \\
\hline Rumination & & & 0.423 & $7.833^{\star \star \star}$ & 0.570 & $7.358^{\star \star \star}$ \\
\hline Anxiety & & & & & 0.408 & $4.548^{\star \star \star}$ \\
\hline$R$ & 0.621 & & 0.664 & & 0.780 & \\
\hline$R^{2}$ & 0.386 & & 0.441 & & 0.609 & \\
\hline$F$ & $30.664^{\star \star \star}$ & & $30.631^{\star \star \star}$ & & $50.053^{\star \star \star}$ & \\
\hline
\end{tabular}

MW, mind-wandering; OCS, obsessive-compulsive symptoms. ${ }^{*} p<0.05,{ }^{* *} p<0.01$, ${ }^{* * *} p<0.001$. 


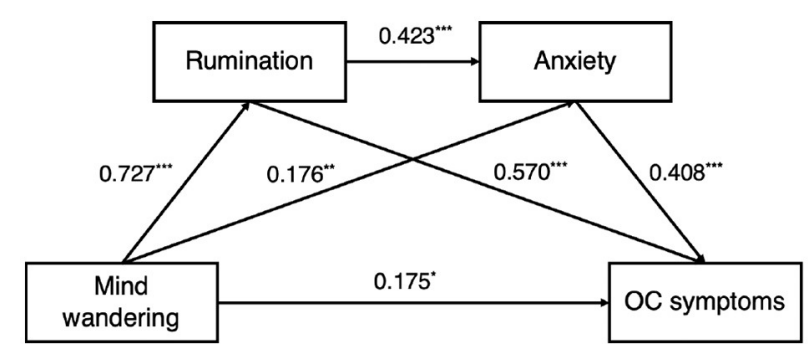

FIGURE 1 | Mediation effect path of rumination and anxiety between mind-wandering and obsessive-compulsive (OC) symptoms. ${ }^{\star \star *} p<0.001$.

TABLE 4 | Hierarchical regression analysis between MW and OCS: Rumination and Depression as mediators.

\begin{tabular}{|c|c|c|c|c|c|c|}
\hline \multirow[t]{2}{*}{ Variables } & \multicolumn{2}{|c|}{ Rumination } & \multicolumn{2}{|c|}{ Depression } & \multicolumn{2}{|c|}{ OCS } \\
\hline & $\beta$ & $t$ & $\beta$ & $t$ & $\beta$ & $t$ \\
\hline Gender & -1.399 & -1.006 & 0.382 & 0.409 & -1.708 & -1.341 \\
\hline Age & -0.105 & -0.893 & 0.051 & 0.652 & 0.338 & $3.145^{\star \star}$ \\
\hline Education & -0.457 & -0.670 & -0.587 & -1.284 & -0.254 & -0.405 \\
\hline MW & 0.727 & $10.766^{\star \star \star}$ & 0.194 & $3.395^{\star \star \star}$ & 0.136 & 1.692 \\
\hline Rumination & & & 0.501 & $10.457^{\star \star \star}$ & 0.453 & $5.544^{\star \star \star}$ \\
\hline Depression & & & & & 0.577 & $5.890^{\star \star \star}$ \\
\hline$R$ & 0.621 & & 0.762 & & 0.796 & \\
\hline$R^{2}$ & 0.386 & & 0.580 & & 0.633 & \\
\hline$F$ & $30.664^{\star \star \star}$ & & $53.580^{\star \star \star}$ & & $55.446^{\star \star \star}$ & \\
\hline
\end{tabular}

MW, mind-wandering; OCS, obsessive-compulsive symptoms. ${ }^{*} p<0.05,{ }^{* \star} p<0.01$, ${ }^{* \star *} p<0.001$.

Results from the mediation effect analysis (see Figure 2) showed that MW did not directly predict OCS (direct effect $=0.136, p=0.092$ ), but rumination and depression fully mediated this relationship (indirect effect $=0.652$ ). Specifically, the mediation effect consisted of three indirect effects:

- Path 1: MW $\rightarrow$ rumination $\rightarrow$ OCS (indirect effect $=0.330$ )

- Path 2: MW $\rightarrow$ depression $\rightarrow$ OCS (indirect effect $=0.112$ )

- Path 3: MW $\rightarrow$ rumination $\rightarrow$ depression $\rightarrow$ OCS (indirect effect $=0.210)$.

None of the bootstrap 95\% confidence intervals contained 0 , indicating that all three paths had a significant indirect effect.

\section{DISCUSSION}

In the present study, OCD patients showed more severe negative affect and abnormal thought activity compared with HCs, which is consistent with previous research findings (49). Further, correlation analysis showed that MW and rumination were significantly and positively correlated with OCS, which is again consistent with previous studies $(6,14)$, suggesting that OCS is closely related to spontaneous thoughts.

MW was conceptualised as the failure of executive control, including a reduced ability to bring one's consciousness back

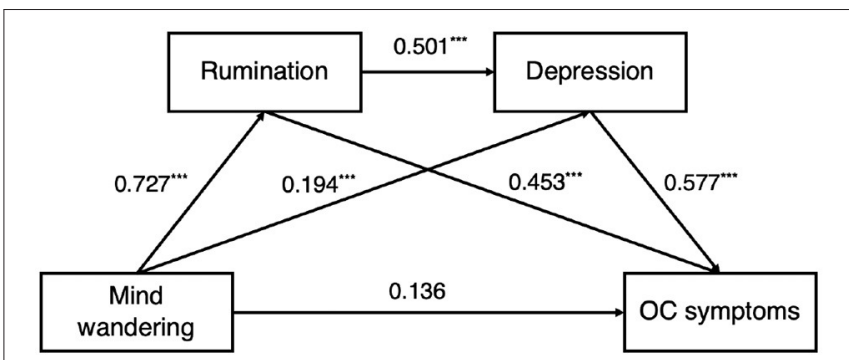

FIGURE 2 | Mediation effect path of rumination and depression between mind-wandering and obsessive-compulsive (OC) symptoms. ${ }^{* \star *} p<0.001$.

to a goal-directed state and enable internally generated taskrelated thoughts to flourish (50). OCD is also characterised by impairments in executive functions (e.g., response inhibition) and sustained attention (51). A recent biological model of differences in executive functioning demonstrated that durable representations in the prefrontal cortex (e.g., a strong image) are associated with weaker prefrontal-posterior connectivity, resulting in impaired inhibition and flexibility in shifting attention (52). This may explain why MW results in decreased integrative functioning and attention deficits (35), implying that MW may impair executive control, in turn inducing and maintaining OCS.

Further, MW and OCS may share a common neurological mechanism. As a mental baseline, MW is considered an outcome of the default mode network (DMN) (53), while OCD has been strongly associated with altered connectivity in the DMN (54). The DMN plays an important role in monitoring the external environment; thus, an abnormal activation of the DMN in OCD may lead to attention lapses and a more internal or external focus. Given this, the findings of this study-the mediating effect of MW on the relationship between clinical OCS and cognitive thought and neurobiological processes-may guide future research.

The mediation analyses conducted in this study highlighted specific factors that may explain the severity of OCS, namely MW, rumination and negative affect. MW had a significant direct effect on OCS; that is, the higher the frequency of MW, the higher the severity of OCS. Further, rumination and negative affect had a chain mediating effect on the relationship between MW and OCS, revealing that the psychological influence of MW on OCS depends on the level of rumination and negative affect.

Notably, MW indirectly influenced OCS through the following three mediation pathways: (i) $\mathrm{MW} \rightarrow$ rumination $\rightarrow$ OCS, (ii) $\mathrm{MW} \rightarrow$ negative affect $\rightarrow$ OCS and (iii) MW $\rightarrow$ rumination $\rightarrow \quad$ negative affect $\rightarrow$ OCS. Individuals who experience MW may focus on internal thoughts, leading to rumination. Negative and catastrophic ruminations on naturally occurring thoughts may lead to compulsive behaviours or neutralisation to suppress these unexpected thoughts and prevent the expected disastrous consequences, exacerbating the severity of OCS.

Given that almost one-third of patients experiencing their first episode of OCD also exhibit symptoms of anxiety and 
depression (55), negative affect may reinforce the occurrence and maintenance of OCS in several ways, including exacerbating functional disability $(56)$, predicting poor quality of life $(49,57)$ and weakening attention control (58). In our assumption model, MW affects OCS not only via rumination but also via negative affect, either directly or indirectly. In particular, rumination and depression play a mediating role in the relationship between MW and OCS severity. Although the relationship between MW and negative affect is still unclear, MW may also result in anxiety or depression in some conditions. MW has been characterised as "coming out of nowhere", resulting in people with OCD feeling threatened or anxious (2). Rumination also results in maladaptive cognition, leading patients to interpret intrusive thoughts in a more negative and catastrophic way and amplifying their distress (59), and inhibits emotional processing (60). This is supported by findings that rumination mediates the relationship between MW and depression (11).

\section{Limitations}

The current study has several limitations. First, while it preliminarily revealed that MW affects OCS via rumination and negative affect, it was unable to specify whether abnormal MW and rumination lead to more severe OC-related beliefs and cognitive distortions in OCD patients. In addition, although we used self-reporting to examine subjects' recent negative affect and spontaneous thoughts, this does not demonstrate cause and effect between spontaneous thoughts and negative affect. Therefore, we suggest that the characteristics of MW during negative emotional states need to be examined. An emotionevoking and experience-sampling paradigm may be used to explore the characteristics of negative affect and spontaneous thoughts and their relationship with OCS. Finally, although PROCESS 3.3 uses the bias-corrected percentile bootstrap method to test for mediation effects and has lower requirements for sample size and data morphology, rendering it suitable for smaller samples, our results should be further validated in a large sample of patients, and longitudinal studies are needed to explore further the causal relationship between MW and OCS.

\section{Conclusion}

This study showed that MW not only has a direct impact on OCS but also increases rumination and negative affect, leading individuals into more negative, catastrophic and uncontrolled

\section{REFERENCES}

1. Patricia V, Cinthia P, Ygor F, Aliende PE, Raony C, Fontenelle LF, et al. Clinical predictors of quality of life in a large sample of adult obsessive-compulsive disorder outpatients. Compr Psychiatry. (2018) 86:82-90. doi: 10.1016/j.comppsych.2018.07.007

2. Fradkin I, Huppert JD. When our train of thought goes off track: The different facets of out-of-context thoughts in obsessive compulsive disorder. J Obsessive Compuls Relat Disord. (2018) 18:31-9. doi: 10.1016/j.jocrd.2018.06.001

3. Lee HJ, Kwon SM. Two different types of obsession: autogenous obsessions and reactive obsessions. Behav Res Ther. (2003) 41:11-29. doi: 10.1016/S0005-7967(01)00101-2 processing of their naturally occurring thoughts, in turn inducing more distressing OCS. These findings contribute to the OCD literature and have both research and clinical implications. Regulating MW and rumination in OCD patients through, for example, mindfulness-based cognitive behaviour therapy may help reduce OCS, abnormal MW activity (61) and negative affect (62).

\section{DATA AVAILABILITY STATEMENT}

The raw data supporting the conclusions of this article will be made available by the authors, without undue reservation.

\section{ETHICS STATEMENT}

The studies involving human participants were reviewed and approved by Research Ethics Committee of Beijing Anding Hospital, Capital Medical University, Beijing, China. The patients/participants provided their written informed consent to participate in this study.

\section{AUTHOR CONTRIBUTIONS}

PW: designed the study, performed literature searches, drafted the manuscript, critically reviewed, and revised the manuscript. WC: performed literature searches, collected dated, and drafted the manuscript. TC: conceptualised the study, reviewed, and revised the manuscript. JG: collected data and revised the manuscript. YL: recruit, assessment, and data collected. XY and FM: both contributed to collected data. ZL and JS: both contribute to conceptualised and designed the study, reviewed, and revised the manuscript, and as co-corresponding authors. All authors contributed to the article and approved the submitted version.

\section{FUNDING}

National Natural Science Foundation of China (No. 81271493). Beijing Municipal Administration of Hospitals Incubating Program (code: PX2020075). Beijing Municipal Administration of Hospitals Clinical Medicine Development of Special Funding Support (code: XMLX202129).
4. Salkovskis PM, Millar JF. Still cognitive after all these years? Perspectives for a cognitive behavioural theory of obsessions and where we are 30 years later Australian. Psychologist. (2016) 51:3-13. doi: 10.1111/ap.12186

5. Salkovskis PM. Understanding and treating obsessive-compulsive disorder. Behaviour Research \& Therapy. (1999) 37:S2952. doi: 10.1016/S0005-7967(99)00049-2

6. Raines AM, Vidaurri DN, Portero AK, Schmidt NB. Associations between rumination and obsessive-compulsive symptom dimensions. Pers Individ Dif. (2017) 113:63-7. doi: 10.1016/j.paid.2017.03.001

7. Christoff K, Irving ZC, Fox KC, Spreng RN, Andrews-Hanna JR. Mindwandering as spontaneous thought: a dynamic framework. Nature Reviews Neuroscience. (2016) 17:718-31. doi: 10.1038/nrn.2016.113 
8. Marchetti I, Koster EHW, De Raedt R. Mindwandering heightens the accessibility of negative relative to positive thought. Conscious Cogn. (2012) 21:1517-25. doi: 10.1016/j.concog.2012.05.013

9. Seli P, Beaty RE, Marty-Dugas J, Smilek D. Depression, anxiety, and stress and the distinction between intentional and unintentional mind wandering. Psychology of Consciousness: Theory, Research, and Practice. (2019) 6:163. doi: $10.1037 / \mathrm{cns} 0000182$

10. Linz R, Pauly R, Smallwood J, Engert V. Mind-wandering content differentially translates from lab to daily life and relates to subjective stress experience. Psychol Res. (2019) 85:649-59. doi: 10.1007/s00426-019-01275-2

11. Marchetti I, Van de Putte E, Koster EH. Self-generated thoughts and depression: from daydreaming to depressive symptoms. Front Hum Neurosci. (2014) 8:131. doi: 10.3389/fnhum.2014.00131

12. Killingsworth MA, Gilbert DT, A. wandering mind is an unhappy mind. Science. (2010) 330:932. doi: 10.1126/science.1192439

13. Callard F, Smallwood J, Golchert J, Margulies D. The era of the wandering mind? Twenty-first century research on self-generated mental activity. Front Psychol. (2013) 4:891. doi: 10.3389/fpsyg.2013.00891

14. Seli P, Risko EF, Purdon C, Smilek D. Intrusive thoughts: Linking spontaneous mind wandering and OCD symptomatology. Psychol Res. (2017) 81:392-8. doi: 10.1007/s00426-016-0756-3

15. Wahl K, Ertle A, Bohne A, Zurowski B, Kordon A. Relations between a ruminative thinking style and obsessive-compulsive symptoms in non-clinical samples. Anxiety, Stress, \& Coping. (2011) 24:217-25. doi: 10.1080/10615806.2010.482985

16. Mahmood Aliloo M, Bakhshipour A, Matinpour B. The role of spontaneous mind-wandering and dispositional mindfulness in predicting obsession symptoms in the college students. Iranian J Psychiat Clinical Psychol. (2018) 24:202-15. doi: 10.32598/ijpcp.24.2.202

17. Audet J-S, Wong SF, Radomsky AS, Aardema F. Not all intrusions are created equal: The role of context, feared-self perceptions and inferential confusion in the occurrence of abnormal intrusions. J Obsessive Compuls Relat Disord. (2020) 26:100537. doi: 10.1016/j.jocrd.2020.100537

18. Marchetti I, Koster EH, Klinger E, Alloy LB. Spontaneous thought and vulnerability to mood disorders: The dark side of the wandering mind. Clin Psychol Sci. (2016) 4:835-57. doi: 10.1177/216770261562 2383

19. Ottaviani C, Shapiro D, Couyoumdjian A. Flexibility as the key for somatic health: From mind wandering to perseverative cognition. Biol Psychol. (2013) 94:38-43. doi: 10.1016/j.biopsycho.2013.05.003

20. Nolen-Hoeksema S, Wisco BE, Lyubomirsky S. Rethinking rumination. Perspectives on Psychological Sci. (2008) 3:400-24. doi: 10.1111/j.1745-6924.2008.00088.x

21. Marchetti I, Koster EHW, De Raedt R. Rest-related dynamics of risk and protective factors for depression: a behavioral study. Clin Psychol Sci. (2013) 1:443-51. doi: $10.1177 / 2167702613489668$

22. Watkins ER. Depressive rumination and co-morbidity: evidence for brooding as a transdiagnostic process. J Rational-Emotive \& Cognitive-Behavior Therapy. (2009) 27:160-75. doi: 10.1007/s10942-009-0098-9

23. Wahl K, van den Hout M, Heinzel CV, Kollárik M, Meyer A, Benoy $\mathrm{C}$, et al. Rumination about obsessive symptoms and mood maintains obsessive-compulsive symptoms and depressed mood: an experimental study. J Abnormal Psychol. (2021) 130:435-42. doi: 10.1037/abn0000677

24. Kollárik $M$, van den Hout $M$, Heinzel CV, Hofer PD, Lieb $\mathrm{R}$, Wahl K. Effects of rumination on unwanted intrusive thoughts: A replication and extension. J Exp Psychopathol. (2020) 11:2043808720912583. doi: 10.1177/2043808720912583

25. Wahl K, van den Hout M, Lieb R. Rumination on unwanted intrusive thoughts affects the urge to neutralize in nonclinical individuals. J Obsessive Compuls Relat Disord. (2019) 20:4-12. doi: 10.1016/j.jocrd.2018.02.002

26. Motivala SJ, Arellano M, Greco RL, Aitken D, Hutcheson N, Tadayonnejad $\mathrm{R}$, et al. Relationships between obsessive-compulsive disorder, depression and functioning before and after exposure and response prevention therapy. Int J Psychiatry Clin Pract. (2018) 22:40-6. doi: 10.1080/13651501.2017.1351991

27. Abramowitz JS, Taylor S, McKay D. Obsessive-compulsive disorder. Lancet. (2009) 374:491-9. doi: 10.1016/S0140-6736(09)60240-3

28. Chen T. Liu L-l, Cui J-f, Qin X-j, Shi H-s, Irish $M$, et al. Balanced Time Perspective and Life Satisfaction: The Mediating
Role of "Temporal Negative Affect". J Happiness Studies. (2020) 22:2563-74. doi: 10.1007/s10902-020-00330-9

29. Watkins ER. Constructive and unconstructive repetitive thought. Psychol Bull. (2008) 134:163-206. doi: 10.1037/0033-2909.134.2.163

30. Rozenman M, Piacentini J, O’Neill J, Bergman RL, Chang S, Peris TS. Improvement in anxiety and depression symptoms following cognitive behavior therapy for pediatric obsessive compulsive disorder. Psychiatry Res. (2019) 276:115-23. doi: 10.1016/j.psychres.2019.04.021

31. Deng Y-Q, Li S, Tang Y-Y. The relationship between wandering mind, depression and mindfulness. Mindfulness.

(2014) 5:124-8. doi: 10.1007/s12671-012-0157-7

32. McLaughlin KA, Nolen-Hoeksema S. Rumination as a transdiagnostic factor in depression and anxiety. Behav Res Ther. (2011) 49:186-93. doi: 10.1016/j.brat.2010.12.006

33. Wilkinson PO, Croudace TJ, Goodyer IM. Rumination, anxiety, depressive symptoms and subsequent depression in adolescents at risk for psychopathology: a longitudinal cohort study. BMC Psychiatry. (2013) 13:250. doi: 10.1186/1471-244X-13-250

34. Smallwood J, O'Connor RC, Heim D. Rumination, dysphoria, and subjective experience. Imagin Cogn Pers. (2005) 24:355-67. doi: 10.2190/AE18-AD1V-YF7L-EKBX

35. Soffer-Dudek N. Dissociative absorption, mind-wandering, and attentiondeficit symptoms: Associations with obsessive-compulsive symptoms. $\mathrm{Br} \mathrm{J}$ Clini Psychol. (2019) 58:51-69. doi: 10.1111/bjc.12186

36. Sheehan DV, Lecrubier Y, Sheehan KH, Amorim P, Janavs J, Weiller E, et al. The Mini-International Neuropsychiatric Interview (M.I.N.I.): the development and validation of a structured diagnostic psychiatric interview for DSM-IV and ICD-10. J Clini Psychiat. (1998) 59:22-33.

37. Goodman WK, Price LH, Rasmussen SA, Mazure C, Delgado P, Heninger GR, et al. The yale-brown obsessive compulsive scale II validity. Arch Gen Psychiatry. (1989) 46:1012-6. doi: 10.1001/archpsyc.1989.01810110054008

38. Foa EB, Huppert JD, Leiberg S, Langner R, Kichic R, Hajcak G, et al. The Obsessive-Compulsive Inventory: development and validation of a short version. Psychol Assess. (2002) 14:485-96. doi: 10.1037/1040-3590.14.4.485

39. Peng ZW, Yang WH, Miao GD, Jing J, Chan RC. The Chinese version of the Obsessive-Compulsive Inventory-Revised scale: replication and extension to non-clinical and clinical individuals with OCD symptoms. BMC Psychiatry. (2011) 11:129. doi: 10.1186/1471-244X-11-129

40. Beck AT, Steer RA. Beck Anxiety Inventory: BAI. San Antonio, TX: Psychological Corporation (1993).

41. Kin-Wing SC, Chee-Wing W, Kit-Ching W, Heung-Chun GC, A. Study of psychometric properties, normative scores and factor structure of beck anxiety inventory Chinese version. Chin J Clini Psychol. (2002) 10:4-6.

42. Sacco R, Santangelo G, Stamenova S, Bisecco A, Bonavita S, Lavorgna L, et al. Psychometric properties and validity of Beck Depression Inventory II in multiple sclerosis. Eur J Neurol. (2016) 23:744-50. doi: 10.1111/ene.12932

43. Wang Z, Yuan C, Huang J. Reliability and validity of the Chinese version of Beck Depression Inventory-II among depression patients. Chin Ment Health J. (2011) 25:476-80.

44. Yang J, Ling Y, Xiao J. The Chinese version of ruminative responses scale in high school students: its reliability and validity. Chin J Clini Psychol. (2009) 17:27-8

45. Qin W. Xiong-zhao Z, Jin-yao Y, Lin C, Ming-jie Z, Yi Z, et al. Reliability and validity of the Chinese version of rumination response scale in depressed patients. J Neurosci Mental Health. (2011) 11:544-6. doi: 10.3969/j.issn.1009-6574.2011.06.002

46. Sheehan PW, Antrobus JS. Daydreaming, imaginal processes, and personality: a normative study. The nature and function of imagery. New York: Academic Press (1972). p. 175-202.

47. Carciofo R, Du F, Song N, Zhang K. Chronotype and time-of-day correlates of mind wandering and related phenomena. Biol Rhythm Res. (2013) 45:37-49. doi: 10.1080/09291016.2013.790651

48. Hayes AF. Introduction to mediation, moderation, and conditional process analysis: A regression-based approach: Guilford Publications (2017).

49. Jahangard L, Fadaei V, Sajadi A, Haghighi M, Ahmadpanah M, Matinnia $\mathrm{N}$, et al. Patients with OCD report lower quality of life after controlling for expert-rated symptoms of depression and anxiety. Psychiatry Res. (2018) 260:318-23. doi: 10.1016/j.psychres.2017.11.080 
50. Smallwood J, Schooler JW. The science of mind wandering: empirically navigating the stream of consciousness. Annu Rev Psychol. (2015) 66:487-518. doi: 10.1146/annurev-psych-010814-015331

51. Abramovitch A, McCormack B, Brunner D, Johnson M, Wofford N. The impact of symptom severity on cognitive function in obsessivecompulsive disorder: A meta-analysis. Clin Psychol Rev. (2019) 67:36-44. doi: 10.1016/j.cpr.2018.09.003

52. Herd SA. O'Reilly RC, Hazy TE, Chatham CH, Brant AM, Friedman NP. A neural network model of individual differences in task switching abilities. Neuropsychologia. (2014) 62:375-89. doi: 10.1016/j.neuropsychologia.2014.04.014

53. Mason MF, Norton MI, Van Horn JD, Wegner DM, Grafton ST, Macrae CN. Wandering minds: the default network and stimulus-independent thought. Science. (2007) 315:393-5. doi: 10.1126/science.1131295

54. Peng ZW, Xu T, He QH, Shi CZ, Wei Z, Miao GD, et al. Default network connectivity as a vulnerability marker for obsessive compulsive disorder. Psychol Med. (2014) 44:1475-84. doi: 10.1017/S0033291713002250

55. Peris TS, Rozenman M, Bergman RL, Chang S, O’Neill J, Piacentini J. Developmental and clinical predictors of comorbidity for youth with obsessive compulsive disorder. J Psychiatr Res. (2017) 93:72-8. doi: 10.1016/j.jpsychires.2017.05.002

56. Storch EA, Abramowitz JS, Keeley M. Correlates and mediators of functional disability in obsessive-compulsive disorder. Depress Anxiety. (2009) 26:806-13. doi: 10.1002/da.20481

57. Kugler BB, Lewin AB, Phares V, Geffken GR, Murphy TK, Storch EA. Quality of life in obsessive-compulsive disorder: the role of mediating variables. Psychiatry Res. (2013) 206:43-9. doi: 10.1016/j.psychres.2012. 10.006

58. Moradi M, Fata L, Ahmadi AA, Abbasi I. Comparing attentional control and intrusive thoughts in obsessive-compulsive disorder, generalized anxiety disorder and non-clinical population. Iranian J Psychiat. (2014) 9(2): 69-75.
59. Wong QJJ, Moulds ML. Impact of rumination versus distraction on anxiety and maladaptive self-beliefs in socially anxious individuals. Behav Res Ther. (2009) 47:861-7. doi: 10.1016/j.brat.2009.06.014

60. Segerstrom SC, Tsao JCI, Alden LE, Craske MG. Worry and rumination: repetitive thought as a concomitant and predictor of negative mood. Cognit Ther Res. (2000) 24:671-88. doi: 10.1023/A:1005587311498

61. Rahl HA, Lindsay EK, Pacilio LE, Brown KW, Creswell JD. Brief mindfulness meditation training reduces mind wandering: the critical role of acceptance. Emotion. (2017) 17:224. doi: 10.1037/emo0000250

62. Wang $\mathrm{Y}, \mathrm{Xu} \mathrm{W}$, Zhuang $\mathrm{C}$, Liu $\mathrm{X}$. Does mind wandering mediate the association between mindfulness and negative mood? A preliminary study Psychological Reports. (2017) 120:118-29. doi: 10.1177/00332941166 86036

Conflict of Interest: The authors declare that the research was conducted in the absence of any commercial or financial relationships that could be construed as a potential conflict of interest.

Publisher's Note: All claims expressed in this article are solely those of the authors and do not necessarily represent those of their affiliated organizations, or those of the publisher, the editors and the reviewers. Any product that may be evaluated in this article, or claim that may be made by its manufacturer, is not guaranteed or endorsed by the publisher.

Copyright (C) 2021 Wang, Cao, Chen, Gao, Liu, Yang, Meng, Sun and Li. This is an open-access article distributed under the terms of the Creative Commons Attribution License (CC BY). The use, distribution or reproduction in other forums is permitted, provided the original author(s) and the copyright owner(s) are credited and that the original publication in this journal is cited, in accordance with accepted academic practice. No use, distribution or reproduction is permitted which does not comply with these terms. 